\title{
Evaluation of In Vitro and In Vivo Flow Rate Dependency of Budesonide/Formoterol Easyhaler ${ }^{\circledR}$
}

\author{
L. Pekka Malmberg, MD, PhD, ${ }^{1}$ Mark L. Everard, MB, ChB, MD, \\ Jussi Haikarainen, MSc(Tech.), ${ }^{3}$ and Satu Lähelmä, MSc(Pharm. ${ }^{4}$
}

\begin{abstract}
Background: The Easyhaler ${ }^{\circledR}$ (EH) device-metered dry powder inhaler containing budesonide and formoterol is being developed for asthma and chronic obstructive pulmonary disease (COPD). As a part of product optimization, a series of in vitro and in vivo studies on flow rate dependency were carried out.

Methods: Inspiratory flow parameters via EH and Symbicort ${ }^{\circledR}$ Turbuhaler ${ }^{\circledR}$ (TH) inhalers were evaluated in 187 patients with asthma and COPD. The $10^{\text {th }}, 50^{\text {th }}$, and $90^{\text {th }}$ percentile flow rates achieved by patients were utilized to study in vitro flow rate dependency of budesonide/formoterol EH and Symbicort TH. In addition, an exploratory pharmacokinetic study on pulmonary deposition of active substances for budesonide/formoterol $\mathrm{EH}$ in healthy volunteers was performed.

Results: Mean inspiratory flow rates through $\mathrm{EH}$ were 64 and 56 L/min in asthmatics and COPD patients, and through TH 79 and $72 \mathrm{~L} / \mathrm{min}$, respectively. Children with asthma had marginally lower PIF values than the adults. The inspiratory volumes were similar in all groups between the inhalers. Using weighted $10^{\text {th }}, 50^{\text {th }}$, and $90^{\text {th }}$ percentile flows the in vitro delivered doses (DDs) and fine particle doses (FPDs) for EH were rather independent of flow as $98 \%$ of the median flow DDs and $89 \%-93 \%$ of FPDs were delivered already at $10^{\text {th }}$ percentile air flow. Using $\pm 15 \%$ limits, $\mathrm{EH}$ and TH had similar flow rate dependency profiles between $10^{\text {th }}$ and $90^{\text {th }}$ percentile flows. The pharmacokinetic study with budesonide/formoterol $\mathrm{EH}$ in healthy subjects $(n=16)$ revealed a trend for a flow-dependent increase in lung deposition for both budesonide and formoterol.

Conclusions: Comparable in vitro flow rate dependency between budesonide/formoterol EH and Symbicort TH was found using the range of clinically relevant flow rates. The results of the pharmacokinetic study were in accordance with the in vitro results showing only a trend of flow rate-dependant increase in lung deposition of active substances with EH.
\end{abstract}

Key words: dry powder inhaler, flow rate dependency, peak inspiratory flow, Easyhaler

\section{Introduction}

A STHMA AND CHRONIC OBSTRUCTIVE PULMONARY DISA Ease (COPD) are significant public health problems and cause a substantial economic burden on societies. ${ }^{(1-3)}$ In most countries, both diseases are increasing in prevalence, and are predicted to remain leading causes of morbidity and mortality worldwide. ${ }^{(4-6)}$ COPD is currently the fourth leading cause of death globally ${ }^{(7)}$ and it is projected to become the third leading cause by $2020 .^{(8)}$
Inhalation is the preferred route for administration of drugs to patients with asthma and COPD as it facilitates direct exposure of the airways to the therapeutic agents. Inhalation also includes a clinical effect at considerably lower doses than used in oral treatments and thereby the risk of systemic side-effects is reduced. Corticosteroids and adrenergic $\beta_{2}$-agonist bronchodilators are the two major classes of asthma drugs. Most of the agents within the classes have been successfully formulated for inhalation-alone or as a combination in a single inhaler. Inhaled anticholinergics

\footnotetext{
${ }^{1}$ Department of Allergy, Helsinki University Central Hospital, Helsinki, Finland.

${ }^{2}$ Department of Respiratory Medicine, Sheffield Children's Hospital, Sheffield, United Kingdom.

${ }^{3}$ Orion Corporation ORION PHARMA, Espoo, Finland.

${ }^{4}$ Orion Corporation ORION PHARMA, Kuopio, Finland.
} 


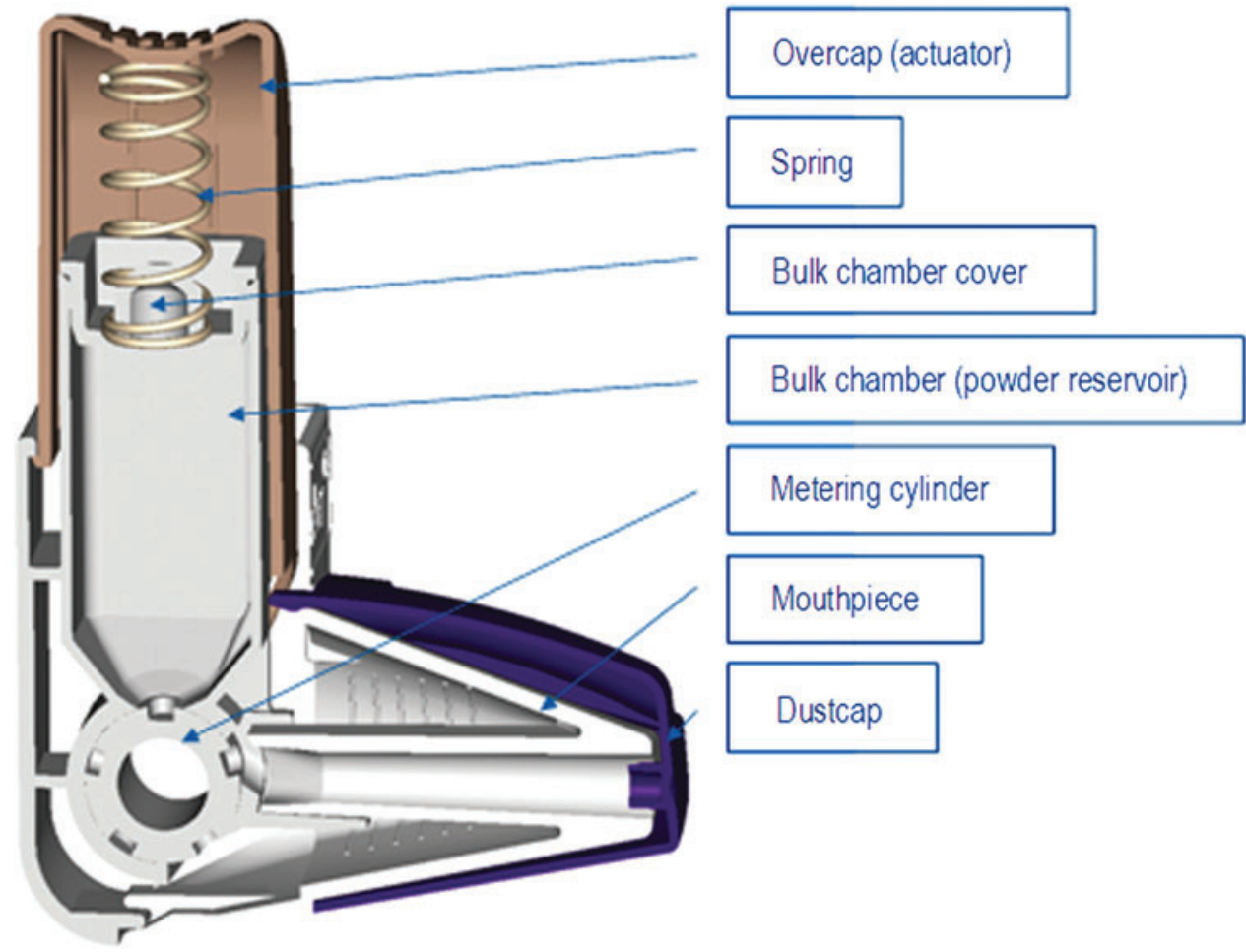

FIG. 1. Cross section of the Easyhaler multi-dose powder inhaler.

have for many years been used for the treatment of COPD. In recent years long-acting inhaled $\beta_{2}$-agonists (LABAs) and inhaled corticosteroids (ICSs) have been approved for treatment and prevention of exacerbations in patients with COPD.

There are a large number of inhalation devices available for drug administration to patients with both asthma and COPD,${ }^{(9-11)}$ but many are associated with significant disadvantages. ${ }^{(12)}$ Nebulizers are useful for children, as they rely only on tidal breathing and do not require a co-ordinated inhalation technique, but they are associated with high costs and inconvenience. Pressurized metered-dose inhalers (pMDIs) provide a convenient method of drug administration at a low cost, but many patients are unable to use them effectively and so they do not receive expected treatment benefits. ${ }^{(13-15)}$ The main problem with the pMDIs is inappropriate inhalation technique-predominantly due to poor coordination of actuation of the inhaler with inhalation. ${ }^{(16)}$ Breath-actuated dry powder inhalers (DPIs) were developed to overcome the coordination problems associated with pMDIs. The DPIs offer advantages as they are compact and free of CFC. ${ }^{(17)}$ At present, however, there are only a few pharmaceutical companies providing a DPI platform with which patients can deliver a range of active substances (either separately or in a combination) at different treatment steps and disease stages.

The Easyhaler ${ }^{\circledR}(\mathrm{EH})$ device-metered dry powder inhaler (dmDPI) (Orion Pharma, Espoo, Finland) has been designed to resemble a conventional pMDI in terms of shape and operating principles, being both simple and practical. ${ }^{(18)}$ It contains powder [active substance(s) in a mixture with lactose] for up to 200 doses. Accurate dosing is achieved by pushing down the overcap of the device that rotates the metering cylinder at the bottom of the bulk chamber (Fig. 1). The dose of drug to be inhaled is positioned in the mouthpiece that is shaped to allow optimal drug distribution to the airways. The dose is inhaled by a simple technique requiring neither co-ordination nor manual dexterity. Currently, salbutamol, beclomethasone, budesonide, and formoterol $\mathrm{EH}$ products are marketed for treatment of asthma (in adults and children $\geq 6$ years of age), and formoterol and salbutamol $\mathrm{EH}$ products also for treatment of COPD. To provide patients with a wide range of active substances in the $\mathrm{EH}$ inhaler, a fixed combination product containing budesonide and formoterol fumarate dihydrate is being developed for the treatment of both asthma and COPD.

Since dmDPIs are driven by inhalation, their performance is dependent upon the inspiratory flow produced by the patient and the properties of the inhaler. ${ }^{(19)}$ This means that the force required to generate the respirable dose emitted during inhalation is dependent on inspiratory flow parameters and the resistance within the device. In the development of a second entry orally inhaled product, budesonide/formoterol $\mathrm{EH}$, characterization of flow rate dependency and comparison to the originator budesonide/formoterol product (Symbicort $^{\circledR}$ Turbuhaler $^{\circledR}$ (TH), AstraZeneca, Södertälje, Sweden) ${ }^{(20)}$ was an important step of the program. One of the main targets of the development work was to demonstrate comparable flow rate dependency between the products within the range of clinically relevant flow rates to apply demonstrated therapeutic equivalence to the patient population covered by the authorization for the originator, as suggested in the EMA guideline for orally inhaled products (CPMP/EWP/4151/00 Rev 1). ${ }^{(21)}$

Here we report a series of in vitro and in vivo flow rate dependency investigations with budesonide/formoterol $\mathrm{EH}$. 
First, the inspiratory flow parameters of patients with asthma (including children, adults, and elderly) and COPD were characterized via the $\mathrm{EH}$ and $\mathrm{TH}$ inhalers. The results of this study determined the minimum, median, and maximum achievable peak inspiratory flow (PIF) rates through the inhalers in the intended patient population. These data were then used for the required in vitro testing to investigate the aerosol characteristics of the products. Next, an assessment of the flow rate dependency of the pharmacokinetic parameters following administration via $\mathrm{EH}$ was conducted in healthy volunteers to explore how relevant the in vitro testing is with regard to in vivo flow rate dependency.

\section{Material and Methods}

Three distinct studies were conducted: first, inspiratory flow parameters were characterized using the budesonide/ formoterol $\mathrm{EH}$ and Symbicort $\mathrm{TH}$ inhalers in patients with asthma and COPD; second, in vitro testing to investigate the flow deposition characteristics of the products were carried out; and third, budesonide and formoterol pharmacokinetic parameters were assessed in healthy subjects following delivery using the $\mathrm{EH}$ inhaler with different PIF rates during administration. The in vivo studies were approved by the local Ethics Committee (EC) and were conducted in accordance with the principles of the Declaration of Helsinki. All participants provided written informed consent prior to participation in any study-specific procedures.

In the first study, inspiratory flow parameters were assessed using the EH and Symbicort TH inhalers (no active treatment administered) in asthmatic children, adolescents, adults, and elderly patients, as well as in patients with COPD of all ages. This open, randomized, crossover study was conducted at four centers in Finland and in the UK. To be included in the study, subjects had to be $\geq 6$ years old with a documented diagnosis of asthma and/or COPD, verified according to GINA and GOLD guidelines, across a range of different severities. Subjects were excluded if they suffered any severe chronic respiratory disease other than asthma or COPD, had an acute respiratory infection, or were unable to perform the necessary spirometric measurements. As part of screening assessments, spirometry was carried out according to American Thoracic Society/European Respiratory Society (ATS/ERS) guidelines including the recording of native PIF rate, ${ }^{(22)}$ height and weight were measured, and demographic data recorded. On the morning of the study day, subjects had to withhold the use of their inhaled medication. Short-acting sympathomimetics were not to be used for at least 8 hours before spirometry and inspiratory flow measurements through inhalers.

The patient received inhaler-specific training (according to the instructions for use of each inhaler). After a practice period, three inspiratory flow curves were recorded as described earlier ${ }^{(23)}$ through the inhalers connected to a pneumotachograph (Spiromaster MX, Medikro Ltd, Kuopio, Finland) in the standing position; the best of the three measurements was analyzed (i.e., the curve with the highest PIF rate). The PIF rate was the primary variable, and inspiratory volume was recorded at the same time as a secondary variable. Training and inspiratory flow parameter measurements were performed in random order. As the outcome of this study, the minimum $\left(10^{\text {th }}\right.$ percentile $)$, median $\left(50^{\text {th }}\right.$ percentile $)$, and maximum $\left(90^{\text {th }}\right.$ percentile $)$ flow rates achieved by the patients were determined.

The in vitro flow rate dependency was assessed by using similar flow rates achieved by the patients of the first study (i.e., the minimum, median, and maximum flow rates were used for delivered dose (DD) and fine particle dose (FPD) measurements). FPD was the mass of particles under $5 \mu \mathrm{m}$. $\mathrm{DD}$ and FPD were determined according to the in vitro testing of DPIs established by the European Pharmacopoeia monograph Preparations for Inhalation ${ }^{(24)}$ using Next Generation Impactor (NGI, apparatus E). The cut-off points for impactor stages were calculated in relation to the used flow rates according to European Pharmacopoeia, and FPD was derived from the data. The number of batches included was 1-2 for EHs and 3 for TH. The in vitro studies were performed by Medfiles Oy, Kuopio, Finland.

The healthy volunteer study comprised an exploratory, open, randomized, crossover, single dose pharmacokinetic study conducted at the Clinical Pharmacology Unit, Orion Pharma, Espoo, Finland. Subjects were required to be 18-55 years old, in good health, have an $\mathrm{FEV}_{1}$ at least $80 \%$ of the predicted value measured at screening, and to be able to inhale the study drug with the required PIF rates. Exclusion criteria included any evidence of clinically significant disease, a need for regular concomitant treatment, or a history of vasovagal collapses or anaphylactic reactions. The subjects received a single dose of two inhalations of budesonide/formoterol EH 320/9 $\mu \mathrm{g} /$ inhalation (i.e., 640/18 $\mu \mathrm{g}$ ) during three treatment periods separated by a wash-out period of 3-21 days. After overnight fasting, the study drug was administered with concomitant oral charcoal (Carbomix, Leiras Takeda, Helsinki, Finland) to prevent absorption via the gastrointestinal tract (GI) and to assess absorption via the lungs only (pulmonary deposition). The amount and dosing regimen of charcoal were previously shown to block GI absorption of the same orally administered dose of budesonide and formoterol efficiently (at least 98.8\%, data on file). Study subjects were trained to perform the administration according to the EH instructions for use (breathe in forcefully and deeply) but either with a target PIF rate (treatments $\mathrm{A}$ and $\mathrm{B}$ ) or without a target PIF rate (treatment $\mathrm{C}$ ). The administration technique was checked before each study drug administration.

- Treatment A: administration with a target PIF rate corresponding to a flow rate attained by the $10^{\text {th }}$ percentile of the intended patient population

- Treatment B: administration with a target PIF rate corresponding to a $4 \mathrm{kPa}$ pressure drop across the inhaler

- Treatment $\mathrm{C}$ : administration without a target PIF rate (with subject's maximum PIF rate)

During study drug administration, PIF rates through the EH connected to a pneumotachograph (Spiromaster MX, Medikro Ltd, Kuopio, Finland) were recorded, and if the administration was unsuccessful, blood samples were not collected but the period was re-scheduled (treatments A and B). The maximum number of re-scheduled periods was two per subject.

Blood samples for budesonide and formoterol analysis were drawn before drug administration and up to $12 \mathrm{~h}$ for budesonide and up to $24 \mathrm{~h}$ for formoterol after drug 
administration. Budesonide and formoterol concentrations in plasma were determined by separate, validated liquid chromatography-tandem mass spectrometry (LC-MS/MS) methods at PPD, Madison, WI. The lower limit of quantification (LLOQ) for the budesonide method P4290.01 (25) was $10 \mathrm{pg} / \mathrm{mL}$ and for the formoterol method P860.02 ${ }^{(26)}$ $0.5 \mathrm{pg} / \mathrm{mL}$. The bioanalytical analyses were performed according to the principles of applicable good laboratory practice (GLP) and good clinical practice (GCP). The maximum observed concentration of concentration-time curve $\left(\mathrm{C}_{\max }\right)$ and the area under the concentration-time curve from zero to the last sample with quantifiable concentration $\left(\mathrm{AUC}_{\mathrm{t}}\right)$ were the primary pharmacokinetic parameters derived from the concentration-time data. They were calculated by noncompartmental method using WinNonlin $^{\circledR}$ 5.0.1 (Pharsight Corporation, Mountain View, CA, USA) computer program.

Safety and tolerability of different treatments were evaluated based on adverse events (AEs) from the time that a study subject signed the informed consent until the end-ofstudy visit. Other safety assessments (clinical and laboratory) were taken at screening and at the end-of-study primarily for subjects' safety.

It should be noted that the $\mathrm{EH}$ inhaler used comprised the final inhaler version in all studies. In in vitro testing a developmental and the final powder formulation were evaluated, and the pharmacokinetic results were obtained with the developmental powder formulation. The same batch of the developmental formulation was used in the in vitro evaluation and the pharmacokinetic study. The highest strength of the products was studied [i.e., budesonide/formoterol 320/ $9 \mu \mathrm{g}$ per inhalation (delivered dose)].

\section{Statistical analysis}

In the PIF rate study, at least 180 patients were planned to be included and 16 in the healthy volunteer study. Due to the design of the in vivo studies, the sample sizes were not based on any formal power calculations and no formal statistical hypotheses were pre-specified. For the study in asthmatic and COPD patients, the primary variable of PIF rate through the inhalers was analyzed by tabulating descriptive statistics for the asthma and COPD groups, and also for the subgroup of asthmatic children. Mean, median, minimum, and maximum values, standard deviation, and percentage points were computed.

In the in vitro study, means and standard deviations were computed for DDs and FPDs attained with different flow rates. To make the flow rate dependency comparisons feasible, the average DD and FPD results with minimum and maximum flow rates were divided by the corresponding results measured using the median flow rate. Percentages deviating from 100 indicate relative air flow dependency. The relative flow dependency of $\mathrm{EH}$ products (developmental and final formulation) compared to Symbicort forte $\mathrm{TH}$ was evaluated against $\pm 15 \%$ limits.

In the healthy volunteer study, the comparison between the treatments $\mathrm{A}, \mathrm{B}$, and $\mathrm{C}$ was based on the descriptive statistics and inferential statistical methods were considered as explorative analysis. For the comparison between treatments $\mathrm{A}$ and $\mathrm{B}$, and treatments $\mathrm{B}$ and $\mathrm{C}$, a general linear mixed model for budesonide and formoterol $\mathrm{C}_{\max }$ and $\mathrm{AUC}_{\mathrm{t}}$ was applied. $90 \%$ confidence intervals (CIs) for the ratio of the geometric means were calculated. These CIs were evaluated against the bioequivalence $(\mathrm{BE})$ region from 0.80 to 1.25 .

All the statistical analyses were performed by or under the supervision of the Department of Biostatistics and Data Management of Orion Pharma. Statistical analyses were performed with $\mathrm{SAS}^{\circledR}$ for Windows (SAS Institute Inc., Cary, NC, USA).

\section{Results}

\section{PIF rate in asthmatic and COPD patients}

In the study of asthmatic and COPD patients to determine clinically relevant inspiratory flow rates, a total of 181 out of the 187 randomized patients had no major protocol deviations such as use of restricted concomitant medication or assessments not taken per-protocol, and so were included in the per-protocol (PP) data set used in the analysis. Of these, 137 patients had asthma and 44 patients had COPD, while the subpopulation of children (from 6 to 11 years) with asthma comprised 52 patients (Table 1). Subjects in both

Table 1. Demographic Characteristics in Asthmatic and COPD Patients and Separately for Subgroup of Asthmatic Children in PIF Rate Study (Intention-to-Treat Data Set)

\begin{tabular}{|c|c|c|c|}
\hline Variable & $\begin{array}{l}\text { Asthma } \\
\mathrm{N}=143\end{array}$ & $\begin{array}{l}C O P D \\
\mathrm{~N}=44\end{array}$ & $\begin{array}{c}\text { Asthmatic children } \\
\mathrm{N}=54\end{array}$ \\
\hline Age in years, median (range) & $38.0(6-82)$ & $67.0(47-80)$ & $9.0(6-11)$ \\
\hline Height in $\mathrm{cm}$, median (range) & $158.0(117-188)$ & $162.5(148-183)$ & $132.5(117-154)$ \\
\hline Weight in kg, median (range) & $62.0(19-117)$ & $70.0(42-140)$ & $30.0(19-63)$ \\
\hline BMI, $\mathrm{kg} / \mathrm{m}^{2}$, median (range) & $22.8(13-51)$ & $26.7(15-43)$ & $17.3(13-30)$ \\
\hline $\mathrm{FEV}_{1}$ in liters, median (range) & $2.1(0.5-5.0)$ & $1.3(0.5-3.3)$ & $1.8(0.5-2.8)$ \\
\hline $\mathrm{FEV}_{1}$ as $\%$ of predicted value, median (range) & $90(35-164)$ & $52(18-92)$ & $93(35-132)$ \\
\hline \multicolumn{4}{|l|}{ Sex, $n(\%)$} \\
\hline Female & $82(57.3)$ & $25(56.8)$ & $33(61.1)$ \\
\hline Male & $61(42.7)$ & $19(43.2)$ & $21(38.9)$ \\
\hline \multicolumn{4}{|l|}{ Race, $n(\%)$} \\
\hline Caucasian & $141(98.6)$ & $44(100.0)$ & $52(96.3)$ \\
\hline Asian & $1(0.7)$ & $0(0.0)$ & $1(1.9)$ \\
\hline Other & $1(0.7)$ & $0(0.0)$ & 1 (1.9) \\
\hline
\end{tabular}


Table 2. Mean PIF Rate (L/Min) and Inspiratory Volume Through Easyhaler

and Turbuhaler Inhalers in Asthmatic and COPD Patients and Separately for Subgroup of Asthmatic Children (Per-Protocol Data Set)

\begin{tabular}{lccc}
\hline Inspiratory parameter & $\begin{array}{c}\text { Patients with asthma } \\
\mathrm{N}=137\end{array}$ & $\begin{array}{c}\text { Patients with COPD } \\
\mathrm{N}=44\end{array}$ & $\begin{array}{c}\text { Asthmatic children } \\
\mathrm{N}=52\end{array}$ \\
\hline $\begin{array}{l}\text { PIF rate, mean (SD) } \\
\text { Easyhaler }\end{array}$ & $63.7(11.5)$ & & \\
$\quad$ Turbuhaler & $79.4(14.4)$ & $75.8(13.0)$ & $61.0(11.1)$ \\
Inspiratory volume (L), mean (SD) & & & $76.5(14.1)$ \\
$\quad$ Easyhaler & $1.8(0.7)$ & $1.6(0.6)$ & $1.4(0.4)$ \\
$\quad$ Turbuhaler & $1.9(0.7)$ & $1.8(0.6)$ & $1.5(0.5)$ \\
\hline
\end{tabular}

disease groups were predominantly female. In the subgroup of asthmatic children the mean age was 8.7 years. Nearly all subjects $(98.4 \%)$ were using medication for their asthma and/or COPD at the time of the study and the ventilatory function at the time of the study varied from severely reduced to normal (Table 1).

As expected, the PIF rates measured through $\mathrm{EH}$ were lower than those measured through Symbicort TH (Table 2) because of the higher internal resistance of EH. For EH, $4 \mathrm{kPa}$ pressure drop across the inhaler results in approximately $55 \mathrm{~L} / \mathrm{min}$ flow whereas for Symbicort TH the flow is approximately $62 \mathrm{~L} / \mathrm{min}$, respectively, for $\mathrm{EH}$ the airflow resistance is $69 \mathrm{~Pa}^{0.5} \cdot \mathrm{s} / \mathrm{L}$ and for Symbicort TH $61 \mathrm{~Pa}^{0.5} \cdot \mathrm{s} / \mathrm{L}$. The mean PIF rates were slightly lower in asthmatic children ( $<12$ years) than in the asthma group as a whole for both EH and TH inhalers. The COPD patients had the lowest mean PIF rates. In the asthmatic patients, the PIF rate ranged from 35.4 to $101.4 \mathrm{~L} / \mathrm{min}$ and in the COPD patients from 27.0 to $83.7 \mathrm{~L} / \mathrm{min}$ for $\mathrm{EH}$ (Fig. 2). The range of PIF rates for $\mathrm{TH}$ was from 48.4 to $123.6 \mathrm{~L} / \mathrm{min}$ and from 35.6 to $102.1 \mathrm{~L} / \mathrm{min}$ in asthmatic and COPD patients, respectively. There was a high correlation between different inhalations (coefficient of correlation above 0.92 for all comparisons, Fig. 3) and the estimated coefficient of reliability was 0.95 for TH and 0.93 for $\mathrm{EH}$.

With respect to the inspiratory volume, the mean values varied between 1.6 and $1.9 \mathrm{~L}$ in asthmatic and COPD patients. Asthmatic children were able to inhale a mean volume of $1.4 \mathrm{~L}$ via $\mathrm{EH}$ and $1.5 \mathrm{~L}$ via $\mathrm{TH}$.

To provide PIF rates for in vitro testing purposes, the achieved PIF rates through the EH and TH inhalers by the $10^{\text {th }}, 50^{\text {th }}$, and $90^{\text {th }}$ percentiles (i.e., minimum, median, and maximum achievable PIF rates) were weighted with the estimated proportion of asthmatic and COPD patients using LABA and ICS combinations in Europe during 2012..$^{(27,28)}$ This was because the proportion of patients in the study did not represent the proportion of patients in the population to whom the product will be indicated. According to the results, the $10^{\text {th }}$ percentile of the intended target population would achieve a PIF rate of $44.7 \mathrm{~L} / \mathrm{min}$ using the $\mathrm{EH}$, with a PIF rate achievable by $50 \%$ of the population being $61.1 \mathrm{~L} / \mathrm{min}$ and by $90 \%$ of the population $74.8 \mathrm{~L} / \mathrm{min}$. Correspondingly, $10^{\text {th }}, 50^{\text {th }}$, and $90^{\text {th }}$ percentile PIF rates for TH would be 54.6, 78.6, and $93.4 \mathrm{~L} / \mathrm{min}$, respectively. The intended target population would achieve lower PIF rates than those achieved by the study subjects on average. This is explained by COPD patients achieving lower PIF rates than asthmatics.
The proportion of COPD patients in this study was lower than their estimated proportion in the intended target population.

\section{In vitro flow rate dependency}

Comparison of the in vitro flow rate performance of budesonide/formoterol EHs and Symbicort forte TH was carried out for their relative DD and FPD values between the extreme and median flows. For Easyhaler, the in vitro performance over the specified air flow range is rather independent of the air flow as $98 \%$ of median flow DDs and $89 \%-93 \%$ of the median flow FPDs are delivered already at $10^{\text {th }}$ percentile air flow (Table 3). For Turbuhaler, the DDs are slightly more air flow dependant between $10^{\text {th }}$ percentile, and median flow as $87 \%$ and $88 \%$ of the median flow DDs are delivered at $10^{\text {th }}$ percentile air flow. The FPDs of both inhalers are less dependent on the air flow between the median flow and $90^{\text {th }}$ percentile flow than with lower flows. In the in vitro comparison using $\pm 15 \%$ limits, both $\mathrm{EHs}$ and TH have similar air flow dependency profile between $10^{\text {th }}$, $50^{\text {th }}$, and $90^{\text {th }}$ percentile flows (Fig. 4). The developmental and final Easyhaler formulations have the similar flow rate dependency characteristics and no remarkable differences can be noted between them.

\section{Pharmacokinetic study in healthy volunteers}

In the healthy volunteer study to determine in vivo flow rate dependency, 18 subjects were screened and 16 of these were entered in the study; two subjects failed to meet the study inclusion criteria. There were no premature discontinuations from the study, thus all the 16 subjects completed the study. All study subjects were Caucasian with a mean age of 25 years (range: $20-35$ years), and half of all subjects were male and half female.

Although subjects practiced the inhalation target flows, the target PIF rates for treatments A and B were difficult to attain and 17 periods in total were re-scheduled. Regardless of repeated attempts, four subjects had to be excluded from the PP analysis. The realized mean PIF rate values were within the target range for treatments $\mathrm{A}$ and $\mathrm{B}$, and $82.0 \mathrm{~L} / \mathrm{min}$ for treatment C (Table 4).

A trend for a flow rate dependent increase in lung deposition was seen for both budesonide and formoterol. The mean $\mathrm{C}_{\max }$ and $\mathrm{AUC}_{\mathrm{t}}$ were the lowest when the PIF rate during administration was the lowest (treatment A) and highest with the highest PIF rate (treatment C), with the trend seeming to be slightly stronger for formoterol than for 
FIG. 2. Individual PIF rates (L/min) by age for Easyhaler (a) and Tubuhaler (b) inhalers in asthmatic and COPD patients $(N=181)$.
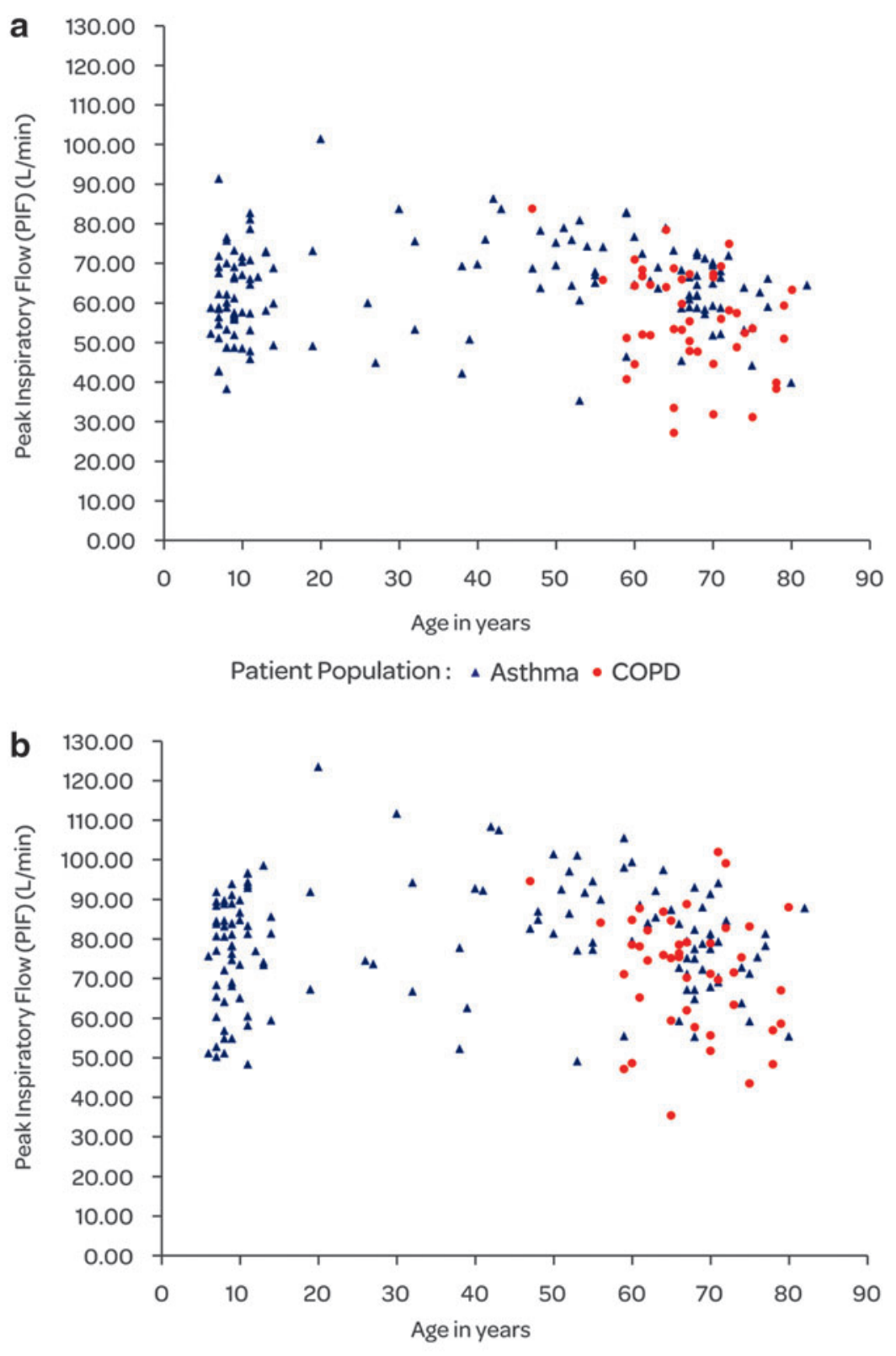

Patient Population: ^ Asthma • COPD budesonide (Fig. 5, Table 5). However, the differences between the treatments were not statistically significant. In the $\mathrm{BE}$ analysis between treatments $\mathrm{A}$ and $\mathrm{B}$, all other ratio estimates and their $90 \%$ CIs were within the classical $\mathrm{BE}$ acceptance range except for the lower limit CI of formoterol $\mathrm{AUC}_{\mathrm{t}}$ (Fig. 6). When the mean PIF rate during drug administration increased from 56.4 to $82.0 \mathrm{~L} / \mathrm{min}$ (treatments $\mathrm{B}$ and $\mathrm{C}$ ), the ratio estimates for $\mathrm{C}_{\max }$ and $\mathrm{AUC}_{\mathrm{t}}$ comparisons were still between 0.80 to 1.25 for both active substances but 3 out of 4 CIs exceeded the lower BE limit.

Safety of the study treatments was good as was expected based on the long experience with the active substances and the dose administered being within the recommended dose range. A total of 61 events were reported by 13 subjects $(81.3 \%)$ during the study with approximately similar incidence between the treatments. The most common AE was headache (33 events), followed by palpitations (5), and cough (3). For the majority of the events reported the severity was assessed as mild. There were no serious AEs.

\section{Discussion}

Inhalation is the preferred route of administration of drugs for treatment of airway diseases, and many different inhalation devices have been developed. However, it is obvious that all patients, including children, elderly, and patients with very poor lung function or severe airway obstruction, cannot use all inhalation devices optimally. It is also obvious that there is no inhaler on the market that is perfect for everybody. ${ }^{(29,30)}$ It is therefore important that physicians choose and recommend a device that suites the individual patient, ${ }^{(17,31)}$ and patient preferences and satisfaction have also to be acknowledged. ${ }^{(17)}$ Each inhaler has its own 
a

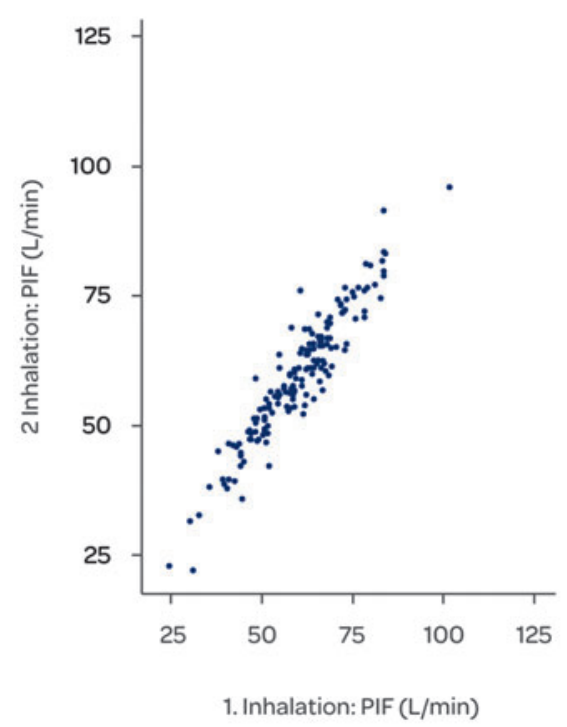

b

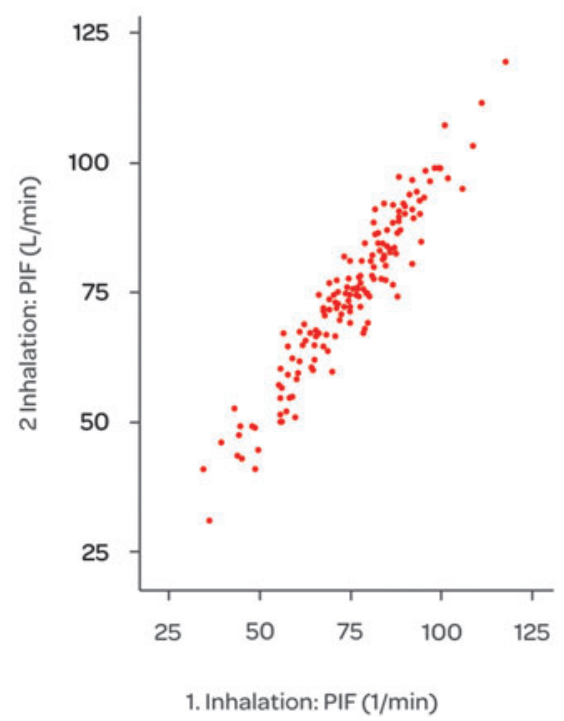

Easyhaler

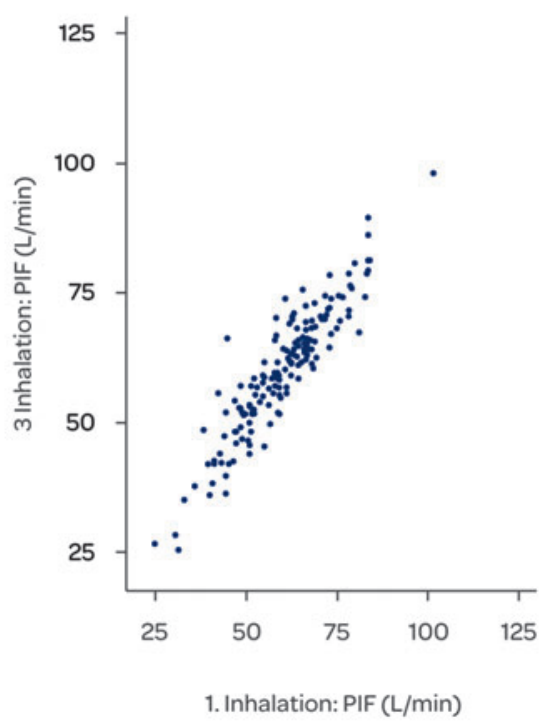

Turbuhaler

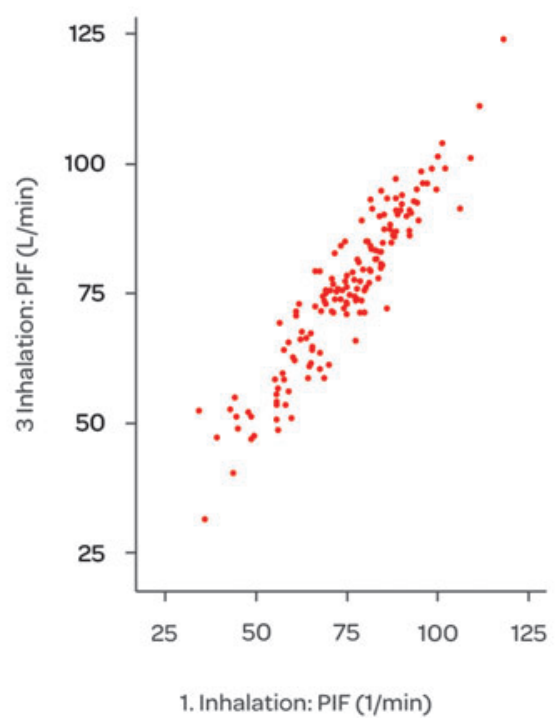

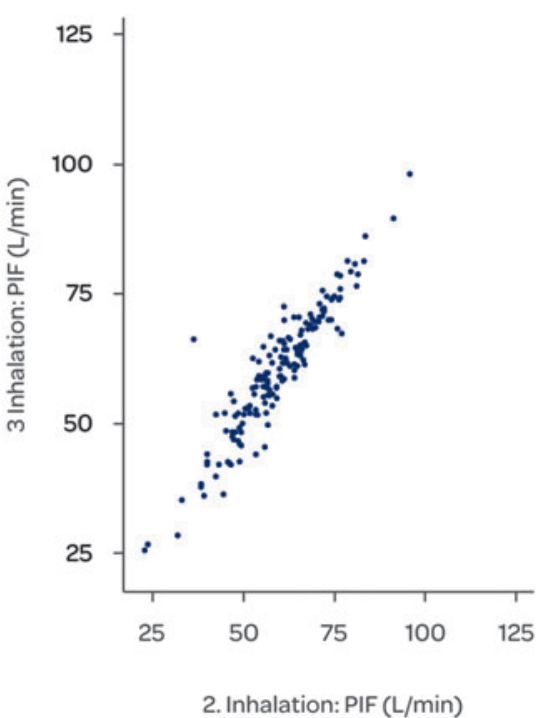

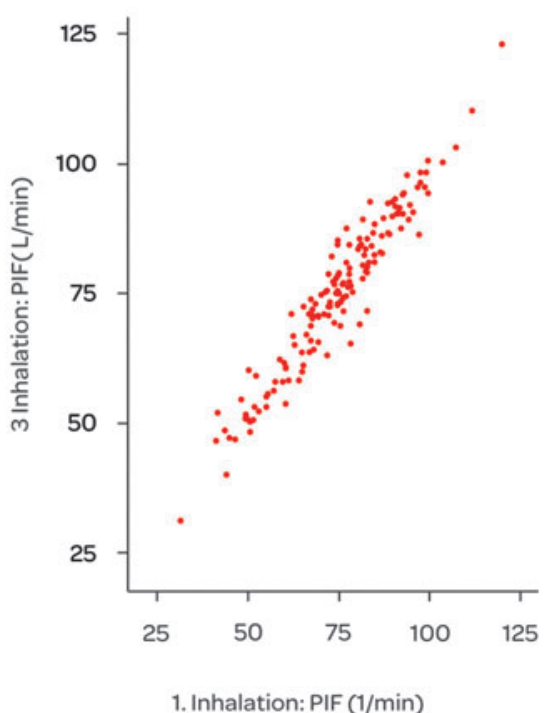

FIG. 3. Scatterplots of PIF rates (L/min) during the different inhalations for Easyhaler (a) and Turbuhaler (b) in PIF rate study.

instructions for optimal use. Therefore, it is confusing when a patient has to use simultaneously two or more different inhalers with different instruction for each of them. It should be a clear advantage if all drugs to be inhaled could be administered via the same inhaler with easy and identical instructions for use. ${ }^{(32)}$

To provide asthma and COPD patients with a reliable inhaler platform with which several active substances can be administered, a new product with EH dmDPI was developed. One of the main targets of the development work with the budesonide/formoterol combination was to demonstrate comparable in vitro flow rate dependency to the reference budesonide/formoterol product, Symbicort TH. However, the current European regulatory environment is challenging with fairly new guidelines (CPMP/EWP/4151/00 Rev 1) ${ }^{(21)}$ and only few second entry products have been approved so far.
This article reports the investigation of inspiratory flow parameters via $\mathrm{EH}$ and $\mathrm{TH}$ inhalers in the intended patient population of LABA/ICS combination product users, in vitro testing to determine products' flow deposition characteristics, and an investigation on pulmonary deposition as an effect of different PIF rates after administration of budesonide/formoterol EH.

The study on PIF rates had a sufficient number of patients with a wide age and severity range of both diseases. As expected, the PIF rates measured through EH were lower than the ones measured through TH because of the higher internal resistance of EH. It is well known that with low resistance the attained PIF rates increase and this is true of all DPIs. ${ }^{(33)}$ The inspiratory volumes were more or less identical with the two inhalers. The COPD patients had the lowest flow rates via both inhalers, not children as usually 
Table 3. Relative DD and FPD (\%) Over Patient Flow Rate Range for Budesonide/Formoterol EASyhaler 320/9 $\mu \mathrm{G} /$ INHalation AND Symbicort Forte TurbuhaleR

\begin{tabular}{|c|c|c|c|c|c|c|c|c|c|}
\hline \multirow[b]{2}{*}{ Product } & \multirow[b]{2}{*}{ Flow* } & \multicolumn{2}{|c|}{ Budesonide } & \multicolumn{2}{|c|}{ Formoterol } & \multicolumn{2}{|c|}{ Budesonide } & \multicolumn{2}{|c|}{ Formoterol } \\
\hline & & $D D$ & $R S D$ & $D D$ & $R S D$ & $F P D$ & $R S D$ & $F P D$ & $R S D$ \\
\hline \multirow[t]{3}{*}{ Easyhaler developmental formulation } & Min & 98 & 9.3 & 98 & 10.9 & 93 & 1.1 & 91 & 2.9 \\
\hline & Median & 100 & 8.4 & 100 & 9.1 & 100 & 0.4 & 100 & 1.1 \\
\hline & Max & 98 & 9.6 & 99 & 7.7 & 102 & 4.4 & 105 & 4.1 \\
\hline \multirow[t]{3}{*}{ Easyhaler final formulation } & Min & 98 & 8.1 & 98 & 8.9 & 89 & 7.2 & 90 & 6.8 \\
\hline & Median & 100 & 8.0 & 100 & 8.7 & 100 & 3.0 & 100 & 1.3 \\
\hline & Max & 103 & 9.4 & 102 & 9.7 & 102 & 5.1 & 101 & 4.1 \\
\hline \multirow[t]{3}{*}{ Symbicort forte Turbuhaler } & Min & 87 & 13.8 & 88 & 13.8 & 86 & 10.2 & 86 & 10.5 \\
\hline & Median & 100 & 18.9 & 100 & 18.8 & 100 & 8.5 & 100 & 8.5 \\
\hline & Max & 97 & 14.2 & 97 & 14.5 & 100 & 8.9 & 101 & 8.9 \\
\hline
\end{tabular}

*Weighted PIF rates through the EH and TH inhalers achieved by the $10^{\text {th }}(\mathrm{min}), 50^{\text {th }}$ (median), and $90^{\text {th }}(\max )$ percentiles of asthmatic and COPD patients.
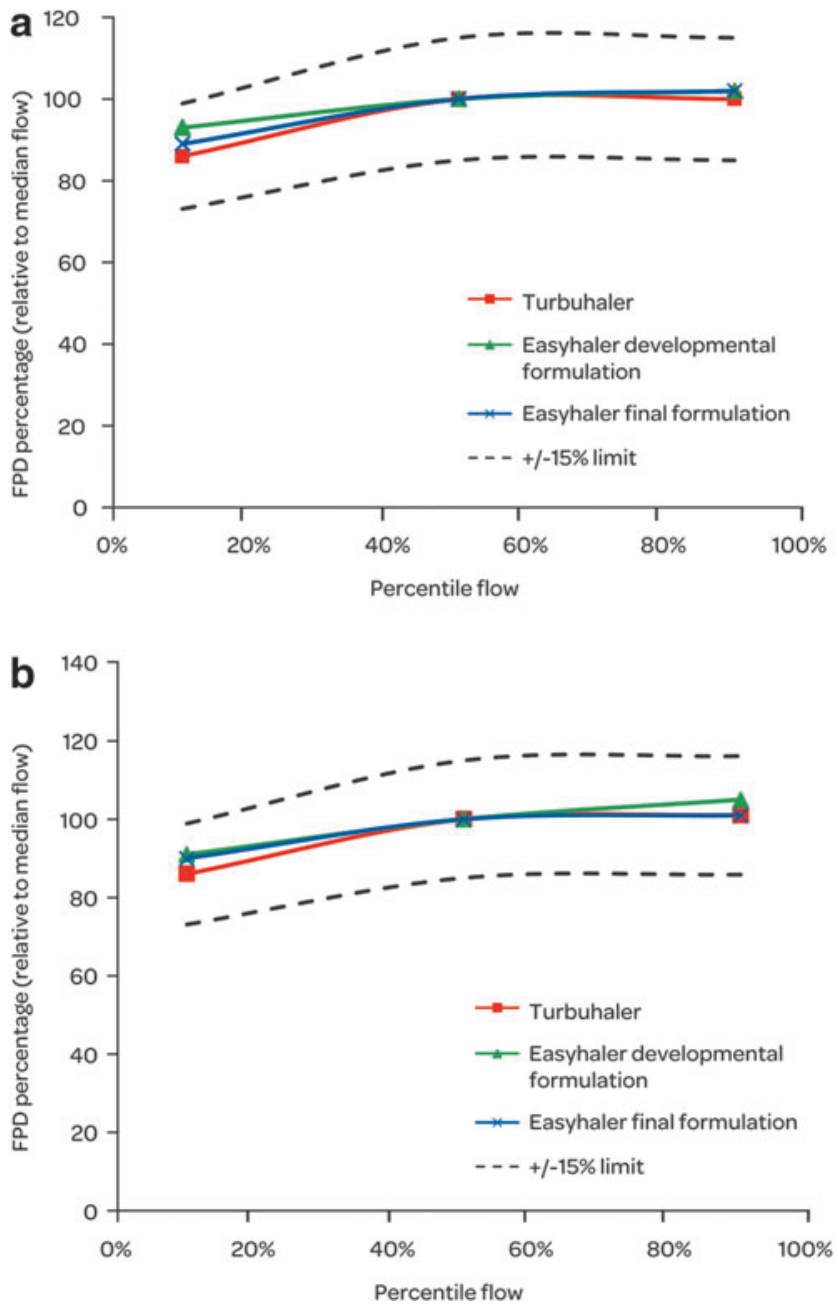

FIG. 4. Relative in-vitro flow rate dependency of budesonide (a) and formoterol (b) FPD on $10^{\text {th }}, 50^{\text {th }}$, and $90^{\text {th }}$ percentile PIF rates for budesonide/formoterol Easyhaler 320/9 $\mu \mathrm{g} /$ inhalation (developmental and final formulation) and Symbicort forte Turbuhaler. thought. Age is inversely related to maximal inspiratory flows, ${ }^{(23)}$ and this probably contributes to the lower flow rates of COPD patients. The results are in line with previously published results with both $\mathrm{EH}^{(23,34)}$ and $\mathrm{TH}^{(35,36)}$ and confirmed that both inhalers can be effectively used by most patients with airway diseases.

In the PIF rate study, the number of patients with COPD was low compared to their proportion in the population to whom the product will be indicated. Therefore, the PIF rates had to be weighed in order to provide PIF rates relevant to target population for in vitro testing. The PIF rates through the $\mathrm{EH}$ and $\mathrm{TH}$ inhalers by the $10^{\text {th }}, 50^{\text {th }}$, and $90^{\text {th }}$ percentiles (i.e., minimum, median, and maximum achievable PIF rates) were weighted with the estimated proportion of asthma and COPD patients using LABA and ICS combinations in Europe in 2012. ${ }^{(27,28)}$ Such weighted averages are analogous to standardized rates of the frequency of occurrence of health phenomena commonly used in epidemiology. It has been estimated that, in 2012, $62 \%$ of the patients using LABA/ISC combination were asthmatics and $38 \%$ were COPD patients. ${ }^{(27,28)}$

The highest PIF rate value of each patient was used in the analysis. That has been the standard practice in similar studies assessing PIF rates via different inhalers. ${ }^{(37-41)}$ Our study aimed to find a typical flow rate that is achievable for each patient. By selecting the highest value, exceptionally low PIF rate values (e.g., due to unsuccessful inhalation) were excluded. However, there was good reproducibility of PIF rate from one inhalation to another. The PIF rates that are typical for the patients in daily life may be difficult to predict by the lung disease or anthropometric factors, since numerous other factors such as training, adherence/compliance, and cooperation, ${ }^{(13,17,37)}$ which are beyond this and similar experimental studies, play an important role as well.

The relative flow rate dependency of EH products (developmental and final formulation) compared to Symbicort forte $\mathrm{TH}$ was evaluated against $\pm 15 \%$ limits, which are general in vitro equivalence requirement in EMA guideline for clinical documentation for orally inhaled products and were therefore applied also in our in vitro study. ${ }^{(21)} \mathrm{TH}$ showed slightly higher flow dependency for DDs between $10^{\text {th }}$ percentile and median flows than EH. Also the relative standard deviation highlights a slightly higher variability for DDs with the $\mathrm{TH}$ inhaler. At the $50^{\text {th }}$ and $90^{\text {th }}$ percentile 
Table 4. PIF Rates (L/Min) Measured during Study Treatment Administrations in the Pharmacokinetic Study (Per-Protocol Data Set)

\begin{tabular}{lcccc}
\hline Treatment & $\mathrm{n}$ & Mean PIF rate $(S D)$ & Min-Max & Target PIF rate \\
\hline A (10 $0^{\text {th }}$ percentile PIF rate) & 12 & $45.5(2.4)$ & $40.2-48.7$ & $43.0-47.0$ \\
B (4 kPa PIF rate) & 12 & $56.4(3.8)$ & $50.9-61.7$ & $53.0-59.0$ \\
C (without target PIF rate) & 12 & $82.0(9.8)$ & $57.2-99.2$ & - \\
\hline
\end{tabular}

flows, only minor differences are seen between the inhalers and between the two EH formulations. For EH formulations, this was an expected result as they both were late phase formulations, the most notable difference being approximately $5 \%$ difference in budesonide concentration of the powder. Overall, no differences in DDs or FPDs were seen between the two $\mathrm{EH}$ formulations and $\mathrm{TH}$; the products had similar flow rate dependency profiles with clinically relevant flow rates. Based on the results both EH formulations could have been carried forward in the development of budesonide/formoterol $\mathrm{EH}$ product.

It should be noted that the volume used in the in vitro study was $4 \mathrm{~L}$ (according to pharmacopeia), i.e., higher than that seen in patients. Nevertheless, patients have sufficient volumes as the doses typically leave reservoir type of DPIs quickly and flow acceleration at the start of inhalation is more important in terms of aerosol dispersion than the actual flow or volume achieved. ${ }^{(35,42)}$ The way to improve in vitro comparison and make it clinically more relevant would be to compare inhalers at relevant inspiratory efforts and to use actual inhalation profiles from patients ${ }^{(43)}$ and realistic mouth-throat models in testing. ${ }^{(44)}$

It has been shown in in vitro studies that the dose emitted from some, if not most, DPIs is dependent on flow at least to some extent. ${ }^{(45)}$ However, in vitro flow rate dependency should be considered significant only if it leads to clinically relevant differences in efficacy or safety responses between administrations at different flows. The
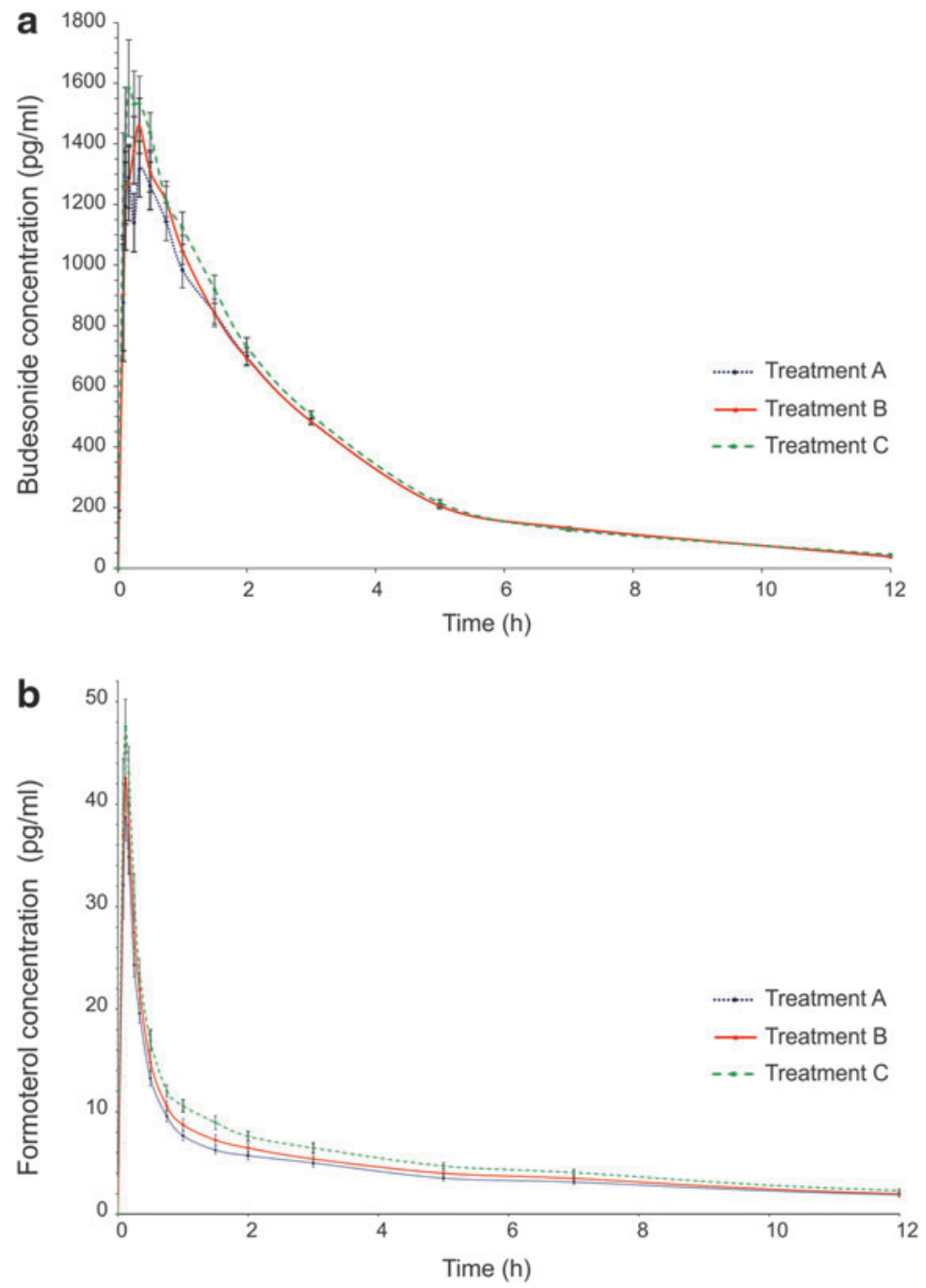

FIG. 5. Budesonide (a) and formoterol (b) concentrations in plasma $(\mathrm{pg} / \mathrm{mL})$ after two inhalations of budesonide/formoterol Easyhaler 320/ $9 \mu \mathrm{g} /$ inhalation with concomitant charcoal administration (mean, SE). Treatment A: administration with a $10^{\text {th }}$ percentile target PIF rate; Treatment B: administration with a $4 \mathrm{kPa}$ target PIF rate; Treatment $\mathrm{C}$ : administration with subject's maximum PIF rate. 
Table 5. $\mathrm{C}_{\text {max }}$ and AUC $\mathrm{T}_{\mathrm{T}}$ alues for Budesonide and Formoterol (Estimated Geometric Means and 90\% CIs) And Coefficient of Variation (CV\%) in Pharmacokinetic Study $(\mathrm{N}=12)$

\begin{tabular}{|c|c|c|c|c|c|c|c|c|}
\hline \multirow[b]{2}{*}{ Treatment } & \multicolumn{4}{|c|}{ Budesonide } & \multicolumn{4}{|c|}{ Formoterol } \\
\hline & $C_{\max }(p g / m L)$ & $C V \%$ & $A U C_{t}(p g \times h / m L)$ & $C V \%$ & $C_{\max }(p g / m L)$ & $C V \%$ & $A U C_{t}(p g \times h / m L)$ & $C V \%$ \\
\hline A & $1522(1272-1821)$ & 34 & $3912(3533-4331)$ & 21 & $38.5(33.9-43.9)$ & 25 & $65.9(56.8-76.6)$ & 26 \\
\hline $\mathrm{B}$ & 1599 (1336-1912) & 32 & $4072(3678-4509)$ & 15 & $40.8(35.8-46.4)$ & 21 & $74.4(64.0-86.0)$ & 21 \\
\hline $\mathrm{C}$ & $1865(1559-2231)$ & 32 & $4128(3729-4571)$ & 20 & $47.1(41.4-53.6)$ & 24 & $86.4(74.4-100.4)$ & 28 \\
\hline
\end{tabular}

CV\% calculated based on raw data summary statistics.

performed pharmacokinetic study was exploratory, but we wanted to obtain in vivo data on flow dependency property of EH (with developmental formulation) to see how well they conform to previously obtained in vitro results.

The PIF rates of the in vitro and in vivo studies differ slightly, with the exception of the $10^{\text {th }}$ percentile target flow. In vitro, the $10^{\text {th }}, 50^{\text {th }}$, and $90^{\text {th }}$ percentile flow rates were 45,61 , and $75 \mathrm{~L} / \mathrm{min}$, respectively; while in vivo the average $10^{\text {th }}$ percentile flow rate, a flow target at $4 \mathrm{kPa}$ pressure drop and no target PIF rate comprise 46, 56, and $82 \mathrm{~L} / \mathrm{min}$ flows. The differences between the realized flows are small, and as
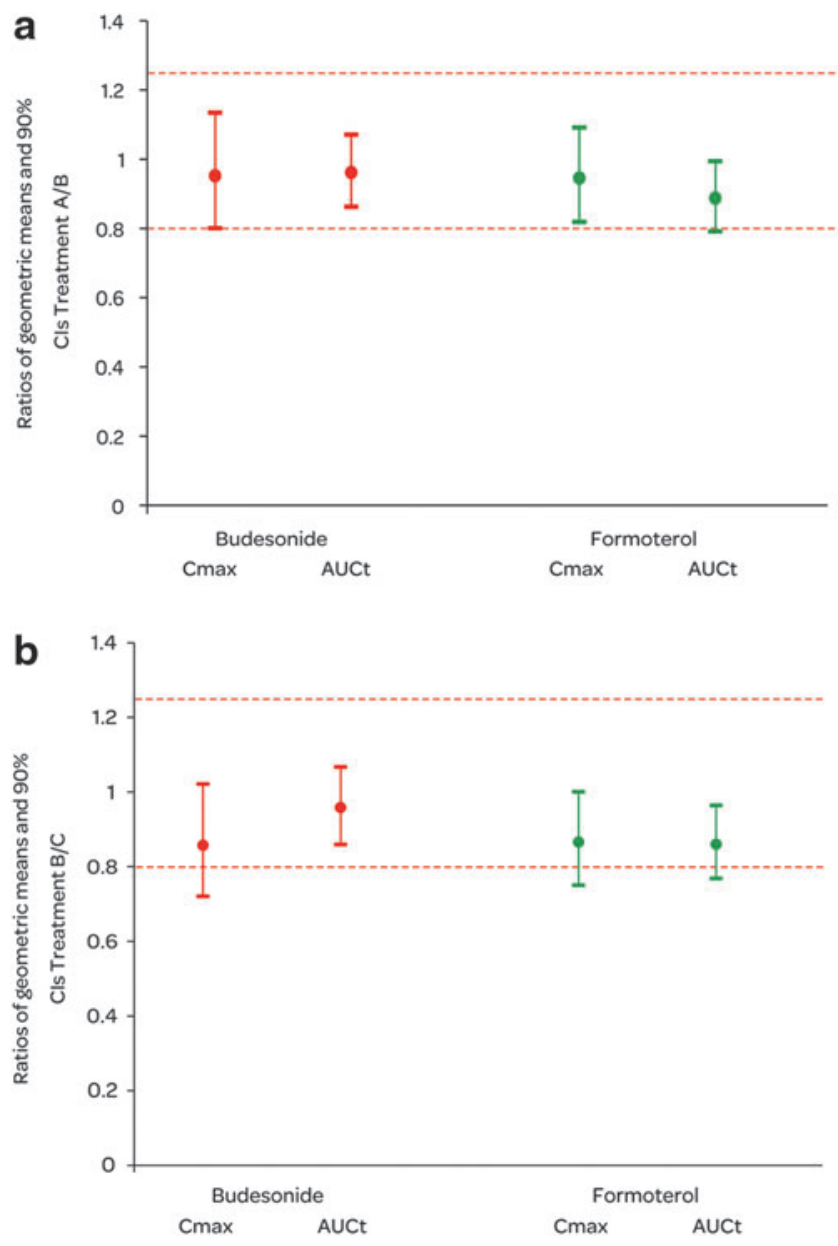

FIG. 6. Summary of budesonide and formoterol $\mathrm{C}_{\max }$ and $\mathrm{AUC}_{\mathrm{t}}$ comparisons between budesonide/formoterol Easyhaler treatments A and B (a) and treatments B and C (b) in the pharmacokinetic study. $N=12$. such, in vitro flow rate dependency testing is relevant with regard to in vivo flow rate dependency.

For the pharmacokinetic study, healthy volunteers were selected as pharmacokinetic testing of orally inhaled products would be more sensitive in healthy volunteers compared with studies in asthmatic patient. This is because of higher pulmonary absorption and less variability in pharmacokinetic parameters. ${ }^{(46-48)}$ Study in healthy volunteers would therefore reveal possible differences in inhaler or formulation performances, and in this case between administration with different PIF rates, with a lower number of subjects. The subjects were trained to perform the administration according to the $\mathrm{EH}$ instructions for use (breathe in forcefully and deeply). In the study, they inhaled at a low flow (treatment $\mathrm{A}$ ), with a flow rate corresponding to a pressure drop of $4 \mathrm{kPa}$ (treatment $\mathrm{B}$ ), and without any flow rate limitations (treatment $\mathrm{C}$ ). Because lung deposition at lower flows was of special interest, the target PIF rates for treatments A and B were close to each other and the criteria for successful administration were rigid. For healthy subjects, the target PIF rates were also low compared to their own achievable maximum and therefore flows were frequently above the targets. Due to these reasons, there were unsuccessful administrations despite the option of rescheduling study periods for up to a maximum of twice per subject. It should be noted that only the subjects with successful administrations were included to the analysis.

In the pharmacokinetic study, a tendency towards higher $\mathrm{C}_{\text {max }}$ and greater $\mathrm{AUC}_{\mathrm{t}}$ was observed with budesonide with increasing flow rates. However, there were no statistically significant differences between the $\mathrm{C}_{\max }$ and $\mathrm{AUC}_{\mathrm{t}}$ values at the lowest flow and those achieved with the subjects' maximum inspiratory flow. Bioequivalent lung deposition was shown for budesonide when $\mathrm{EH}$ was administered at low and $4 \mathrm{kPa}$ PIF rates. For formoterol $\mathrm{AUC}_{\mathrm{t}}$, the lower $90 \% \mathrm{CI}$ marginally exceeded the 0.8 limit. With higher flows (between $4 \mathrm{kPa}$ and maximum PIF rate) budesonide $\mathrm{AUC}_{\mathrm{t}}$ showed bioequivalent lung deposition but for the other parameters, the result was inconclusive (i.e., the CIs have an overlap with the acceptance range).

We have chosen to use BE testing for the evaluation of in vivo flow rate dependency of $\mathrm{EH}$ in an explorative $\mathrm{PK}$ study. BE testing as defined by the European guidance ${ }^{(21)}$ is not applicable as such to flow rate dependency comparisons, but similarity between a second entry and an originator product should be demonstrated with appropriate in vitro data.

The study was a pilot scale study, only 12 subjects were included in the PP data set. The variability of the pharmacokinetic parameters was, however, low and of similar 
magnitude between the treatments, indicating that the study methodology and performance were well standardized. The coefficient of variation $(\mathrm{CV})$ in the budesonide part of the study appears to be similar as in another study with $\mathrm{TH},{ }^{(49)}$ but clearly smaller than in studies with pMDIs. ${ }^{(50)}$ Had the variability remained the same, a study with a sample size of approximately 60 subjects would have shown BE (also CIs completely within the acceptance range) in both comparisons (treatment A vs. B and treatment B vs. C).

Based on the results, it can be concluded that comparable in vitro flow rate dependency between budesonide/formoterol $\mathrm{EH}$ and budesonide/formoterol $\mathrm{TH}$ was demonstrated (within $\pm 15 \%$ limits) using the range of clinically relevant flow rates. The results of the pharmacokinetic study were in accordance with the in vitro results showing only a trend of flow rate dependant increase in lung deposition of active substances with EH.

\section{Acknowledgments}

Orion Pharma funded these studies. We are grateful to Leena Mattila for the conduct of the pharmacokinetic study (principal investigator), Mikko Vahteristo for statistical analysis of the data, and Olof Selroos for medical writing support.

\section{Author Disclosure Statement}

PM has no conflicts of interest; ME has received honoraria from Orion for giving talks and attending advisory boards and has assisted with regulatory submissions. JH and SL are employees of Orion Pharma.

\section{References}

1. Global Initiative for Asthma (GINA). National Heart Lung and Blood Institute, National Institutes of Health. GINA report. Global strategy for asthma management and prevention. Bethesda, NIH Publication Number 02-3659. Updated 2014 at www.ginasthma.com. Accessed June 14, 2014.

2. Global Initiative for Chronic Obstructive Lung Disease (GOLD). National Heart Lung and Blood Institute, National Institutes of Health. GOLD report. Global strategy for diagnosis, management and prevention of COPD. Bethesda, NIH 2009. Updated 2014 at www.goldcopd.org. Accessed June 14, 2014.

3. Sullivan SD, Ramsay SD, and Lee TA: The economic burden of COPD. Chest 2000;11:5S-9S.

4. Lopez AD, and Murray CJ: The global burden of disease, 1990-2020. Nat Med 1998;4:1241-1243.

5. Murray CJ, and Lopez AD: Alternative projections of mortality and disability by cause 1990-2020: Global Burden of Disease Study. Lancet 1997;349:1498-1504.

6. Mathers CD, and Loncar D: Projections of global mortality and burden of disease from 2002 to 2030. PLoS Med 2006; 3:e442.

7. World Health Organization: World health statistics 2008 http://www.who.int/gho/publications/world_health statistics/EN_WHS08. Accessed June 14, 2014.

8. Chapman KR, Mannino DM, Soriano JB, Vermeire PA, Buist AS, Thun MJ, Connell C, Jemal A, Lee TA, Miravitlles M, Aldington S, and Beasley R: Epidemiology and costs of chronic obstructive pulmonary disease. Eur Respir J 2006;27:188-207.
9. Atkins PJ: Dry powder inhalers: An overview. Respir Care 2005;50:1304-1312.

10. Selroos O, Pietinalho A, and Riska H: Delivery devices for inhaled asthma medication. Clinical implications of differences in effectiveness. Clin Immunother 1996;6:273299.

11. Brocklebank D, Ram F, Wright J, Barry P, Cates C, Davies L, Douglas G, Muers M, Smith D, and White J: Comparison of the effectiveness of inhaler devices in asthma and chronic obstructive airway disease; A systematic review of the literature. Health Technol Assess 2001;5:1-149.

12. Laube BL, Janssens HM, de Jongh FHC, Devadason SG, Dhand R, Diot P, Everard ML, Hovarth I, Navalesi P, Voshaar T, and Chryhstyn $\mathrm{H}$ : What the pulmonary specialist should know about the new inhalation therapies. Eur Respir J 2011;37:1308-1331.

13. Lavorini F, Magnan A, Dubus JC, Voshaar T, Corbetta L, Broeders M, Dekhuijzen R, Sanchis J, Viejo JL, Barnes P, Corrigan C, Levy M, and Crompton GK: Effect of incorrect use of dry powder inhalers on management of patients with asthma and COPD. Respir Med 2008;102:593-604.

14. Melani AS, Bonavia M, Cilenti V, Cinti C, Lodi M, Martucci P, Serra M, Scichilone N, Sestini P, Aliani M, and Neri M: Inhaler mishandling remains common in real life and is associated with reduced disease control. Respir Med 2011;105:930-938.

15. Crompton GK, Barnes PJ, Broeders M, Corrigan C, Corbetta L, Dekhuijzen R, Dubus JC, Magnan A, Massone F, Sanchis J, Viejo JL, and Voshaar T: The need to improve inhalation technique in Europe: A report from the Aerosol Drug Management Improvement Team. Respir Med. 2006;100:1479-1494.

16. Crompton GK: Problems patients have using pressurised aerosol inhalers. Eur J Respir Dis 1982;63:101-104.

17. Haughney J, Price D, Barnes NC, Virchow JC, Roche N, and Chrystyn $\mathrm{H}$ : Choosing inhaler devices for people with asthma: Current knowledge and outstanding research needs. Respir Med 2010;104:1237-1245.

18. Silvasti M, Sormunen H, Laurikainen K, Lähelmä S, and Toivanen P: Easyhaler ${ }^{\circledR}$, a novel multidose powder inhalerComparison with metered dose inhaler. Drugs Today 1996; 32:353-363.

19. Palander A, Mattila T, Karhu M, and Muttonen E: In vitro comparison of three salbutamol-containing multidose dry powder inhalers-Buventol Easyhaler ${ }^{\circledR}$, Inspiryl Turbuhaler ${ }^{\circledR}$ and Ventoline Diskus ${ }^{\circledR}$. Clin Drug Invest 2000;20:25-33.

20. Scott LJ: Budesonide/Formoterol Turbuhaler: A review of its use in chronic obstructive pulmonary disease. Drugs 2012;72:395-414.

21. CHMP (2009) Guideline on the requirements for clinical documentation for orally inhaled products (OIP) including the requirements for demonstration of therapeutic equivalence between two inhaled products for use in the treatment of asthma and chronic obstructive pulmonary disease (COPD) in adults and for use in the treatment of asthma in children and adolescents. Available at: http://www .ema.europa.eu/docs/en_GB/document_library/Scientific_ guideline/2009/09/WC500003504.pdf. Accessed June 14, 2014.

22. Miller MR, Hankinson J, and Brusasco V: Standardization of spirometry. ATS/ERS task force: Standardization of lung function testing. Eur Resp J 2005;26:319-338.

23. Malmberg LP, Rytilä P, Happonen P, and Haahtela T. Inspiratory flows through dry powder inhaler in chronic obstructive pulmonary disease: Age and gender rather than 
severity matters. Int J Chron Obstruct Pulmon Dis 2010; 5:257-262.

24. European Pharmacopoeia monograph Preparations for Inhalation http://lib.njutcm.edu.cn/yaodian/ep/EP5.0/07 monographs_on_dosage_forms/Preparations $\% 20$ for $\% 20$ inhalation.pdf. Accessed June 14, 2014.

25. Coaty J, Pierce A, and Swanson J: Analytical method for the determination of budesonide in human $\mathrm{K}_{3}$ EDTA plasma by LC/MS/MS. PPD Method P429.01, May 2010.

26. Wilking M, Zhang ZP, and Zheng N: Analytical method for the determination of formoterol in human lithium heparin plasma by LC/MS/MS. PPD Method P860.02, July 2008.

27. Asthma: Emerging LABA/ICS combinations have a difficult time differentiating themselves from existing agents in the class. Decision Resources, Inc. DecisionBase 2009.

28. Chronic Obstructive Pulmonary Disease: Blockbuster Potential Exists for Biological Drugs That Dramatically Reduce Exacerbation Rates. Decision Resources, Inc. DecisionBase 2009.

29. Chrystyn H: Closer to an 'Ideal Inhaler' with the Easyhaler $^{\circledR}$. An innovative dry powder inhaler. Clin Drug Invest 2006;26:175-178.

30. Borgström L, Asking L, and Thorsson L: Idealhalers or realhalers? A comparison of Diskus and Turbuhaler. Int J Clin Pract 2005;59:1488-1495.

31. Virchow JC, Crompton GK, Dal Negro R, Pedersen S, Magnan A, Seidenberg J, and Barnes PJ: Importance of inhaler devices in the management of airway disease. Respir Med 2008;102:10-19.

32. Price D, Chrystyn H, Kaplan A, Haughney J, RománRodríguez M, Burden A, Chisholm A, Hillyer EV, von Ziegenweidt J, Ali M, and van der Molen T: Effectiveness of same versus mixed asthma inhaler devices: A retrospective observational study in primary care. Allergy Asthma Immunol Res. 2012;4:184-191.

33. Clark A, and Hollingworth A: The relationship between powder inhaler resistance and inspiratory conditions in healthy volunteers: Implications for in vitro testing. J Aerosol Med 1993;6:99-110.

34. Koskela T, Malmström K, Sairanen U, Peltola S, KeskiKarhu J, and Silvasti M: Efficacy of salbutamol via Easyhale ${ }^{\mathrm{r}}{ }^{\circledR}$ unaffected by low inspiratory flow. Respir Med 2000;94:1229-1233.

35. Bisgaard H, Klug B, Sumby B, and Burnell P: Fine particle mass from the Diskus inhaler and Turbuhaler inhaler in children with asthma. Eur Respir Dis 1998;11:1111-1115.

36. Borgström L: On the use of dry powder inhalers in situations perceived as constrained. J Aerosol Med 2001;14: 281-287.

37. Al-Showair RA, Tarsin WY, Assi KH, Pearson SB, and Chrystyn H: Can all patients with COPD use the correct inhalation flow with all inhalers and does training help? Respir Med 2007;101:2395-2401.

38. Baba K, Tanaka H, Nishimura M, Yokoe N, Takahashi D, Yagi T, Yamaguchi E, Maeda Y, Muto T, and Hasegawa T: Age-dependent deterioration of peak inspiratory flow with two kinds of dry powder corticosteroid inhalers (Diskus and Turbuhaler) and relationships with asthma control. J Aerosol Med Pulm Drug Deliv 2011;24:293-301.

39. Janssens W, VandenBrande P, Hardeman E, De Langhe E, Philps T, Troosters T, and Decramer M: Inspiratory flow rates at different levels of resistance in elderly COPD patients. Eur Respir J 2008;31:78-83.
40. Magnussen H, Watz H, Zimmermann I, Macht S, Greguletz R, Falques M, Jarreta D, and Garcia Gil E: Peak inspiratory flow through the Genuair inhaler in patients with moderate or severe COPD. Respir Med. 2009;103:1832-1837.

41. Mahler DA, Waterman LA, and Gifford AH: Prevalence and COPD phenotype for a suboptimal peak inspiratory flow rate against the simulated resistance of the Diskus ${ }^{\circledR}$ dry powder inhaler. J Aerosol Med Pulm Drug Deliv 2013;26:174-179.

42. Azouz W, and Chrystyn H: Clarifying the dilemmas about inhalation techniques for dry powder inhalers: Integrating science with clinical practice. Prim Care Respir J 2012;21: 208-213.

43. Olsson B, and Asking L: Clinical aspects of the function of inspiratory flow driven inhalers. J Aerosol Med 1994;7: S43-S47.

44. Delvadia R, Hindle M, Longest $\mathrm{W}$, and Byron PR: In vitro tests for aerosol deposition II: IVIVs for different dry powder inhalers in normal adults. J Aerosol Med 2013; 26:138-144.

45. Borgström L, Bisgaard H, O'Callaghan C, and Pedersen S. Dry-powder inhalers. In: Bisgaard H, O'Callaghan C, Smaldone GC (eds.) Drug Delivery to the Lung. Lung Biology in Health and Disease Vol. 162. Marcel Dekker, New York/Basel 2002:421-448.

46. Dalby C, Polanowsky T, Larsson T, Borgström L, Edsbäcker S, and Harrison T. The bioavailability and airway clearance of the steroid component of budesonide/formoterol and salmeterol/fluticasone after inhaled administration in patients with COPD and healthy subjects: A randomized controlled trial. Respir Res 2009;10:104.

47. Häussermann S, Acerbi D, Brand P, Herpich C, Poli G, Sommerer K, and Meyer T: Lung deposition of formoterol HFA (Atimos/Forair) in healthy volunters, asthmatic and COPD patients. J Aerosol Med 2007;20:331-341.

48. Mortimer KJ, Harrison TW, Tang Y, Wu K, Lewis S, Sahasranaman S, Hochhaus G, and Tattersfield AE: Plasma concentrations of inhaled corticosteroids in relation to airflow obstruction in asthma. Br J Clin Pharmacol 2006;62:412-419.

49. Persson G, Ankerst J, Gillen M, Bengtsson T, and Thorsson L. Relative systemic availability of budesonide in patients with asthma after inhalation from two dry powder inhalers. Curr Med Res Opin 2008;24:1511-1517.

50. Clearie KL, Williamson PA, Meldrum K, Gillen M, Carlsson LG, Carlholm M, Ekelund J, and Lipworth BJ. Pharmacokinetic and pharmacodynamic comparison of hydrofluoroalkane and chlorofluorocarbon formulations of budesonide. Br J Clin Pharmacol. 2011;71:504-513.

Received on October 3, 2013 in final form, May 20, 2014

Reviewed by: Anthony Hickey Andy Clark Lars Borgström

Address correspondence to: Satu Lähelmä Orion Corporation ORION PHARMA Volttikatu 8, PL 1780 FI-70701, Kuopio Finland

E-mail: satu.lahelma@orionpharma.com 